\title{
Le Manichéisme dans "Le Satyre" de Victor Hugo
}

Quelques critiques ont noté un dualisme dans I'oeuvre de Victor Hugo et ils ont suggéré qu'une influence manichéenne existe dans la philosophie du poète.1 Cependant, autant que nous sachions, aucun entre eux n'a fait une étude plus approfondie sur 1a question du manichéisme dans 1'oeuvre hugolienne. Le but de cette étude est de définir brièvement le manichéisme et de voir à quel point on peut dire que le poème "Le Satyre" dans 1'anthologie La Légende des siècles a été influencé par cette philosohpie dualiste.

Dans son sommaire concis des thèmes principaux du manichêisme, R. McL. Wilson montre que la marque dominante du système de Mani est un dualisme consistant; que ce dualisme refuse la possibilité de tracer les origines du bien et du mal à une seule source; qu'on voit dans la nature $I^{\prime}$ opposition de Dieu et de la matière comme le conflit de la lumière et de 1 'ombre, et comme 1 'opposition de la vérité et de $1^{\prime}$ erreur; que le monde, et $I^{\prime}$ homme en particulier, est un mélange du bien et du mal, le résultat d'une violation des limites originales par les forces du mal.2 Le monde, donc, et 1 'homme en particulier, est devenu un mélange imparfait des forces du bien et des forces du mal. La tâche de 1 'homme est de devenir conscient de la lumière qui réside dans son âme et par cette conscience parfaite d'atteindre le vrai but de la formation de l'univers, la séparation complète du bien et du mal, la séparation de la lumière et de $I^{\prime}$ ombre. Un résultat de ce dualisme, c'est que 1 'homme ". . .n'est plus qu' un champ de bataille entre les forces impersonnelles ou entre les divinités."3

L'examen des cinq divisions du poème nous 
montre très clairement ce mélange de la lumière et de I'ombre. Au début du Prologue ce mélange est très bien décrit par la juxtaposition des mots "nuit" et "jour" et "Vesper" et "Aurore." Le poète nous dépose fermement dans un monde dualiste où la lumière et 1 'ombre sont opposées, mais dans lequel elles doivent coexister. Ensuite, dans la deuxième partie du poème, "Le Bleu," nous nous trouvons avec le Satyre dans le ciel, dans 1 'azur qui est 1 'idéal des poètes symbolistes. On pourrait peut-être croire que l'idéal soit la lumière, le bien. En montant 1 'escalier célèste le Satyre voit "Le ciel, le jour qui monte et qui s'épanouit,/ La terre qui s'efface et 1 'ombre qui se dore."4 Mais une fois arrivé, il voit encore le mal dans "la Force et la Violence" (p.414) des armes des dieux. Nous voyons donc que même chez les dieux il y a un mélange des forces du mal et du bien. Ce mélange est personnifié dans le personnage de Jupiter qui "ressemblait au gouffre où le soleil se plonge"(p. 415). Dans les vers suivants on peut être tenté de croire que le bien et le mal, venant de Jupiter, viennent d'une seule source, principe des philosophies monistes.5 Ou est-ce un mélange du bien et du mal qui se rencontrent dans le personnage de Jupiter?

I1 assignait les sorts; et ses réflexions Etaient gloire aux Cadmus et roue aux Ixions; Sa rêverie, où 1 'ombre affreuse venait faire Des taches de noirceur sur un fond de lumière,

Etait comme la peau du léopard tigré; Selon qu'ils s'écartaient ou s'approchaient,

De ses décisions clémentes ou funèbres, au gré Son pouce et son index faisaient dans les ténèbres S'ouvrir ou se fermer les ciseaux d'Atropos; La radieuse paix naissait de son repos, 
Et la guerre sortait d'un pli de sa narine; (p. 415)

D'abord, 1a mention d'Atropos ici est très importante. Elle est une des trois Parques, déesses grecques qui contrôlent la vie des hommes. Dans notre poème il paraît que Jupiter contrôle les ciseaux d'Atropos mais en effet les trois Parques avaient presqu'autant de pouvoir que lui. 6 De toute façon, nous voyons clairement ici l'opération du destin qui égale pour les manichéens la nécessité. Dans son oeuvre sur le dualisme, Pétrement dit que "1'opposition fondamentale est 1'opposition Nécessité-Liberté." Elle continue, "Le monde est nécessité (Destin, contrainte, esclavage); dans cette nécessité, il y a une semence de liberté qui est d'une autre nature, comme si elle venait d'ailleurs. Mais il faut 1'éveiller, car elle est inconsciente" ( $p$. 133). La nécessité et le destin se trouvent du côté du mal parce qu'ils représentent l'ignorance. Et comme nous verrons plus loin dans cette étude, il est important que 1 'homme échappe au destin dans la connaissance de sa propre origine, c'est-à-dire la lumière (Pét., pp. 175-184).

Jupiter, qui apparaît dans ce passage avec toute sa puissance, ne montre-t-il pas, comme les "archontes" des philosophes gnostiques, "1a puissance de ce qui est aveugle."7 I1 est possible que Hugo suggère cet aveuglement avec sa référence quelques vers plus loin à la "pensée obscure" de Jupiter (p. 415). I1 y a une indication aussi que "l'ombre affreuse" vient d'ailleurs et ne commence pas dans Jupiter. C'est une distinction importante par rapport aux théories manichéennes envers I'origine du bien et du mal, et envers la nature du vrai Dieu. Dans le mythe manichéen, 1 'ombre (comme la lumière) vient d'ailleurs et le vrai Dieu n'est jamais visible. Que Jupiter est très en évidence ici est une indication que dans la pensée de Hugo il n'est 
qu'un "sous-ordre" dans sa conception de 1 'univers. Je crois donc que dans les vers cités ci-dessus, Jupiter représente plutôt un mélange du bien et du mal, au lieu d'en être la source.

On ne peut pas quitter cette partie du poème sans discuter le Zodiaque dont Hugo fait mention. $D^{\prime}$ abord, dans les vers suivants il est évident que le Zodiaque se trouve au-delà de l'olympe.

Au dessus de l'01ympe éclatant, au delà Du nouveau ciel qui naît et du vieux qui croula,

Plus loin que les chaos, prodigieux décom-

Tournait la roue énorme aux douze cages bres, sombres,

Le Zodiaque, ayant autour de ses essieux Douze spectres tordant leur chaîne dans les cieux;

Ouverture du puits de 1 'infini sans borne; (p. 416)

Pour les gnostiques il y avaient entre le monde et le vrai Dieu des barrières constituées par les sphères célestes, c'est-à-dire les sphères des cinq planètes que connaissait l'antiquité, celles du soleil et de la lune et celle des étoiles fixes (Pet., pp. 170-75). Les sept sphères principales avaient des "archontes" comme gardiens. La huitième, l'ogdoade, celle des étoiles fixes, avait les douze signes du zodiaque comme gardiens. Les gardiens étaient nécessaires afin de protéger les portes creusées dans ces voûtes, des portes ("les puits") qui s'ouvrent sur I'autre monde ("I'infini sans borne") où se trouve le vrai Dieu. Ces gardiens ne laissent passer que des âmes pures. On remarque que les dieux mythologiques du poème, certainement à propos de leur place géographique dans l'univers, ressemblent encore une fois plutôt aux "archontes" 
qu'au vrai Dieu qui est toujours au-delà, de l'autre côté des barrières qu'il a construites pour se séparer du monde.

Dans les vers qui suivent la citation ci-dessus nous voyons la lutte primordiale entre les ténèbres (les "douze spectres" qui avaient pour mère "l'âpre Nuit") et la Iumière qui est représentée par Apollon, déité de la lumière dans la mythologie grecque. Dans le mythe manichéen cette bataille tient une place très importante. 8 Mais 1 a version de Hugo diffère dans ce que dans son poème c'est le jour qui commence la bataille tandis que dans le mythe manichéen c'est la force du mal qui la commence. Quoi qu'il en soit, la ressemblance existe; le mythe est le même; qui a commencé la querelle n'est peut-être qu' un point très fin pour un poète. D'ailleurs, Hugo n'est pas connu pour son exactitude en ce qui concerne n'importe quelle philosophie. Enfin le résultat est le même; les gardiens, les signes du zodiaque deviennent des astres. Ils portent désormais la marque de la lumière. Il devient évident que "le bleu" n'est pas trop différent du lieu terrestre où nous avons rencontré le Satyre. Le dualisme du bien et du mal existe dans 1 'un comme dans 1 'autre.

Dans la troisième partie, "Le Noir," le Satyre chante sa chanson de 1a terre. Au début tout est ténèbres mais, comme on verra plus loin, le mélange apparait avant peu de temps. C'est dans cette partie que le Satyre introduit aux dieux le concept de 1 'âme humaine dont 1 a signification sera développée dans la quatrième partie. Dans cette quatrième partie, "Le Sombre," le Satyre chante les difficultés qui naissent du conflit.qu'a produit ce mélange de l'âme humaine avec le mal. C'est la section la plus lyrique du poème et c'est une exhortation de la part du Satyre d'éveiller dans 1 'homme la conscience du pouvoir qui sera le sien avec une réalisation parfaite de sa liberté. Comme dans la philosohpie manichéenne, cette libération n'est pas possible sans une compréhension de la lumière, la puissante force 
du bien qui est contenue dans l'âme humaine.

Enfin dans la dernière partie, "L'Etoile," 1e Satyre révèle qui il est. Il est l'étoile nouvelle qui brille dans le ciel et qui dans le mythe maniché en est "une hérésie dans le ciel, la rupture de l'ordre immuable, quelque chose d'enfin nouveau, d'enfin libre"(Pét., p. 181). On reviendra aux questions du personnage du Satyre et de ses liens avec le manichéisme vers la fin de l'étude, mais on peut constater qu'il y a à travers le poème un dualisme consistant qui semble être influencé par la philosophie manichéenne. Un autre concept important dans le manichéisme, celui de la libération de l'âme humaine est aussi en évidence.

Passons maintenant à 1 'étude du thème de la nature dans le poème. Si on suit la philosophie des manichéens il faut voir la nature comme une partie intégrante de la matière, du monde entier. La matière s'était d'abord opposée au Dieu, elle était l'ignorance et la cause du mal. Mais la doctrine manichéiste change graduellement jusqu'au point où cette perspective pessimiste devient un "dualisme de l'opposition métaphysique de deux principes: lumière et ténèbres, connaissance et ignorance, esprit et matière; et le monde, résultat du mélange, n'est plus mauvais mais imparfait" (Pet., p. 189). Dans la section "Le Noir" du poème, on voit cette même progression. La "terre monstrueuse" avec ses caves, ses trous, ses entonnoirs, des fleuves noirs et des gouffres inouls change à travers le temps.

La racine effrayante. . .

Descend, plonge, atteint $1^{\prime}$ ombre et tâche de la boire,

Et bue, au gré de 1'air, du lieu, de la saison,

L'offre au ciel en encens ou la crache en

Selon que la racine, embaumée ou malsaine

$$
\text { poison, }
$$


Sort, parfum, de 1'amour, ou, vénin, de 1a haine.

(p. 419)

C'est la transition de la nature de son état primordiale où elle existait dans l'ombre, dans l'ignorance, à un nouvel état où le bien et le mal sont opposés. Dans cette chanson passionnée le Satyre décrit la nature comme terrible et effrayante mais enfin il 1 a voit comme un lien entre 1 a matière et Dieu, entre le mal et le bien. Elle est "le lien formidable/ Du bois qui pousse avec l'idéal contemplé!" (p. 421). $\mathrm{Ni}$ arbre ni fleur ne peut pousser sans la lumière du soleil, du ciel. Un lien est ainsi créé entre le ciel et la matière qui est la terre. L'image que crée Hugo ici est très poétique et on peut très bien voir sa conception du rôle de la nature dans un univers dualiste. La nature est imparfaite parce qu'elle forme ce lien entre la matière et le ciel, et comme 1 'homme, elle se trouve dans le conflit entre le mal et le bien.

Dans ce monde imparfait où la nature imparfaite est un champ de bataille, I'homme doit se soumettre à sa propre bataille intérieur qui est aussi un conflit entre le bien et le mal, entre la lumière et les tênèbres. On voit que ce conflit peut devenir extrême, et en effet c'est ce que décrit Hugo: "Et pour 1 'homme qui pense et qui voit, la ciguë" ( $p$. 419). Il nous pose le suicide comme moyen de résoudre le conflit. Mais le suicide est une solution imparfaite ou même pas acceptable, parce qu'en effet c'est une déclaration de 1 'impuissance. La solution pour les manichéens se manifeste dans le personnage d'un sauveur, un "inconnu" qui viendra chercher la perle du monde qui est 1 'âme humaine. Alors 1 'homme n'est pas entièrement abandonné à lutter tout seu1. Ce sauveur viendra du vrai Dieu qui est "au-delà," derrière les barrières qui le séparent des hommes. Le sauveur est inconnu du monde et, en plus, il est inconnu des autres dieux, les "archontes" qui ne savent 
pas qu'il y ait quelqu'un au-dessus d'eux. I1 trompera les autres dieux par sa forme qui sera "la plus pitoyable possible" (Pét., pp. 164-70). Pour le manichéen cet étranger est le Buddha, il est le Christ, il est Zoroastre et enfin il est Mani lui-même.

On remarque un parallelisme qui existe entre le personnage du Satyre et cet étranger, cet "inconnu." Voici le début du poème:

Un satyre habitait 1'olympe, retiré

Dans le grand bois sauvage. . .

Qu'était-ce que ce faune? On 1 'ignorait;

et Flore

Ne le connaissait point, ni Vesper, ni

Qui sait tout. .

1 'Aurore

Personne ne savait le nom de ce maroufle.

(p. 411)

Comme 1 'inconnu des manichéens, même les autres dieux et déesses ne reconnaissent pas la forme de Pan sous son déguisement; ils ne le prennent pas au sérieux à cause de sa forme qui ressemble si peu à celle d'un dieu. Dans la deuxième partie du poème, quand le Satyre est entraîné par Hercule devant les dieux, ceux-ci le méprisent. Jupiter en rit et Vénus tourne son front et demande, "Qu'est-ce que c'est que cette bête-1à?" (p. 416). Mais le Satyre, sachant qui il est, autre signe de I'inconnu manichéen, (Pét., pp. 164-70) ne s'inquiète pas et il va se placer droit devant Vénus. Cette action produit une grande joie dans les autres immortels et ils "riaient du pauvre paysan" (p. 417). Parce qu'ils les a fait rire, Jupiter ne peut pas être fâché contre lui, ni pour la séduction de Psyché (qui est, impossible de l'oublier, la personnification de 1 'âme humaine) ni pour sa bravade devant Vénus, et il lui commande de chanter. Ainsi commence la longue chanson 1yrique dans laquelle le Satyre trace 1 'histoire du monde et la lutte des forces du bien 
et du ma1.

Vers la fin de la troisième partie du poème le Satyre révèle le trésor que 1 'homme contient en lui; c'est 1 'âme humaine, cette même perle que 1 'inconnu des manichéens est venu chercher (Pét., p. 166). Pour le Satyre aussi I'âme est de première importance. Lui aussi, comme 1 'inconnu manichéen, veut trouver I'âme humaine; il veut la révéler à I'homme qui est inconscient du pouvoir de son âme et par cette révélation il veut rendre à la race humaine "son âge d'or." Au même temps il doit éveiller dans 1'homme une conscience du mal parce qu'une réalisation des deux forces est essentielle afin de les distinguer. Il veut montrer à $I^{\prime}$ homme tout le mal qu'il a souffert dans la guerre, sous les rois et aux mains des dieux. On est maintenant droit devant $1^{\prime}$ expression d'un dualisme manichéen.

Seul sur la terre il [1'homme] a la lueur faciale;

Seul il parle; et sans lui tout est décapité. Et 1 'on vit poindre aux yeux du faune la clarté

De deux larmes coulant comme à travers 1a flamme.

Il montra tout le gouffre acharné contre 1 'âme;

Les ténèbres croisant leurs funestes rameaux, Et la forêt du sort et la meute des maux; (p. 425)

Dans les larmes du faune on perçoit la difficulté de sa tâche. Il voit 1'âme dans toute sa beauté, mais elle est toujours obscurcie par le gouffre, les ténèbres et les forces du mal. Tout le conflit du dualisme manichéen se trouve dans ces vers. Ensuite le Satyre devient de plus en plus lyrique et enfin il lance cette exhortation à 1 'homme: "Deviens 1'Humanité, triple, homme, enfant et femme!/ Transfigure-toi! va! sois de plus en plus 1'âme!" (p.427). 
Comme l'inconnu des manichéens, il cherche la libération totale de l'âme pour qu'elle puisse rejoindre la lumière universelle qui est la force du bien. Voilà la fin du poème:

Place à l'atome saint qui brûle ou qui ruisselle!

Place au rayonnement de I'âme universelle! Un roi c'est de la guerre, un dieu c'est de la nuit.

Liberté, vie et foi, sur le dogme détruit! Partout une lumière et partout un génie! Amour! tout s'entendra, tout étant 1 'harmonie!

L'azur du ciel sera l'apaisement des loups Place à Tout! Je suis Pan; Jupiter! à genoux. (p. 430).

Le Satyre se révèle enfin, et il révèle aussi l'énormitê de sa tâche. Il veut déposer tous les rois et tous les dieux afin de permettre au règne de $1^{\prime}$ âme humaine de commencer. Il est l'esprit de la renaissance de 1 'homme.

On peut certainement voir un panthéisme dans ces derniers vers. 9 Et il existe une autre expression très frappante du panthéisme dans 1 a description du métamorphose du Satyre en Pan dans les vers qui précèdent ceux que je viens de citer. Et si on remonte encore un peu plus loin, le Satyre avait chanté, "Je vois. Olmpyes bleus et ténèbreux Avernes,/ Temples, charniers, forêts, cités, aigle, alcyon,/ Sont devant mon regard la même vision;" (p. 428). Ces vers constituent 1 'expression d'un panthéisme plus ou moins orthodoxe. Mais immédiatement après il semble confirmer la vision manichéenne du du vrai Dieu: ". . Quelqu'un est./ Mais celui-1à, jamais 1 'homme ne le connait" (p. 428). Le Dieu manichéen reste toujours inconnu à l'homme. Est-ce donc de lui que chante le Satyre? Encore une considération, c'est que dans la mythologie grecque 
c'est un des dons de Pan de conférer la sagesse.10 Le Satyre transformé en Pan ne fait-il pas la même chose?

A ce point il faut se laisser ouvert à toutes les possibilités. Devant un poète dont l'oeuvre est d'une telle richesse il n'y a pas toujours de conclusion, et on ne peut pas toujours catégoriser toutes ses idées. On voit dans Hugo un exemple extraordinaire de "1'individu" dont parle le passage suivant:

The agony of breaking through personal limitations is the agony of spiritual growth. Art, literature, myth, and cult, philosophy and ascetic disciplines are instruments to help the individual past his limiting horizons into spheres of ever-expanding realization. As he crosses threshold after threshold, conquering dragon after dragon, the stature of the divinity that he summons to his highest wish increases, until it subsumes the cosmos. Finally the mind breaks the bounding sphere of the cosmos to a realization transcending all experience of form--all symbolizations, all divinities: a realization of the ineluctable void.11

Le panthéisme, la mythologie grecque, le manichéisme, et sans doute d'autres que nous n'avons même pas abordés, ils existent tous dans "Le Satyre." II faut aussi considérer le contexte du poème dans $\mathrm{La}$ Légende des siècles et se rendre compte que l'inscription du poème sous le titre "Seizeième Siècle/ Renaissance-Paganisme" est de première importance. Le Satyre-Pan devient la porte-parole de Hugo qui décrit son impression individuelle de la re-naissance de 1 'homme. En l'écrivant, Hugo a utilisé des idées tirées de plusieurs mythologies, religions 
et philosophies; il a ainsi créé son propre mythe de sa vision apocalyptique de la libération de 1 'esprit de 1 'homme après son long sommeil des siècles précédents.

\author{
EILEEN GRABOW \\ UNIVERSITY OF KANSAS
}

\title{
NOTES
}

${ }^{1}$ On peut trouver des références au manichéisme ou au dualisme dans I'oeuvre de Victor Hugo dans les livres suivants: Ch. Renouvier, Victor Hugo le philosophe (Paris: Armand Colin, 1912), pp. 47-122; Auguste Viatte, Victor Hugo et les illuminés de son temps (Montréal: Les Editions de 1'Arbre, 1942), pp. 175-201; Patricia Ward, The Medievalism of Victor Hugo (University Park: Penn. State Univ. Press, 1975), pp. 84-99; Paul Zumthor, Victor Hugo poète de Satan (Paris: Laffont, 1946).

${ }^{2}$ R. McL. Wilson, "Mani and Manichaeism," The Encyclopedia of Philosophy, $1972 \mathrm{ed}$.

${ }^{3}$ Simone Pétrement, Le Dualisme chez Platon, les gnostiques et les manichéens (Vendôme: PUF, 1947), p. 5 .

${ }^{4}$ Victor Hugo, La Légende des siècles, La Fin de Satan, Dieu, ed. Jacques Truchet (Paris: Pléiade, Gallimard, 1950), p. 414. Toutes les citations du poème renvoient à cette édition.

5 Roland Hall, "Monism and Pluralism," The Encyclopedia of Philosophy, 1972 ed. 
6"Fates," The New Columbia Encyclopedia, 1975 ed.

7 Pétrement, p. 178. Les archontes sont les princes ou les dieux de 1a doctrine manichéenne. Leur origine était le sombre, mais après la bataille avec la lumière ils sont devenus un mélange du bien et du mal. Voir R. McL. Wilson et Pétrement pour une description plus détaillée.

8. McL. Wilson; Pétrement, pp. 189-207.

${ }^{9}$ John Porter Houston, Victor Hugo (New York: Twayne Publ. Inc., 1974), p. 124 .

10"Pan," The New Columbia Encyclopedia, 1975 ed.

${ }^{11}$ Joseph Campbe11, The Hero with a Thousand Faces (Princeton: Princeton Univ. Press, 1968), p. 190. 